2018-08

\title{
Tracer advection in a pair of adjacent side-wall cavities, and in a rectangular channel containing two groynes in series
}

\author{
Jalali, MM
}

http://hdl.handle.net/10026.1/17691

10.1007/s42241-018-0064-z

Journal of Hydrodynamics

Springer Science and Business Media LLC

All content in PEARL is protected by copyright law. Author manuscripts are made available in accordance with publisher policies. Please cite only the published version using the details provided on the item record or document. In the absence of an open licence (e.g. Creative Commons), permissions for further reuse of content should be sought from the publisher or author. 


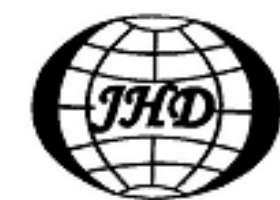

www.sciencedirect.com/ science/journal/10016058

\title{
Tracer advection in a pair of adjacent side-wall cavities, and in a rectangular channel containing two groynes in series
}

\author{
Mohammad Mahdi Jalali ${ }^{1}$, Alistair G.L. Borthwick ${ }^{2}$ \\ ${ }^{1,2}$ Institute for Energy Systems, School of Engineering, The University of Edinburgh, Edinburgh, UK \\ ${ }^{1}$ E-mail: s1370516@ed-alumni.net
}

\begin{abstract}
A model is presented of particle advection near groynes in an open channel. Open channel hydrodynamics is modelled using the shallow water equations, obtained as the depth-averaged form of Reynolds-averaged continuity and Navier-Stokes momentum equations. A Lagrangian particle-tracking model is used to predict trajectories of tracer particles advected by the flow field, with bilinear interpolation representing the continuous flow field. The particle-tracking model is verified for chaotic advection in an alternating flow field of a pair of blinking vortices. The combined shallow flow and Lagrangian particle-tracking model is applied to the simulation of tracer advection in flow past a pair of side-wall cavities separated by a groyne, and in an open rectangular channel containing a pair of parallel groynes oriented normal to the channel wall. The study is potentially useful in understanding mixing processes in shallow flow fields near hydraulic structures in wide rivers,
\end{abstract}

Key words: Shallow water equations; Lagrangian particle tracking; chaotic advection; blinking vortices; side-wall cavities; groynes

\section{Introduction}

Mixing occurs due to advection and dispersion processes whereby species become distributed within a flow field ${ }^{[1]}$. Mixing processes are promoted by shear in a shallow flow primarily through vorticity from eddies, and directly influence the transport of material, heat, and contaminants, thus affecting water quality. In laminar flow, diffusion is entirely due to molecular processes, and follows Fick's Law whereby the rate of mass transfer of a substance per unit area is proportional to the concentration gradient. In turbulent flow, the diffusing substance is transferred by turbulent fluctuations as well as by molecular diffusion, and, by analogy with Fick's First Law, it may be assumed that the turbulent flux is proportional to the gradient of the time-averaged concentration ${ }^{[1]}$. An important factor in river mixing processes is the presence of large-scale turbulent eddies, which may be created from the bed boundary layer as it rolls up into vortices, and from flow separation at obstacles and side wall boundary layers; such eddies play a major role in entraining sediment into suspension and driving sediment transport in a channel. In rivers with erodible beds, the flow hydrodynamics alters the channel morphology and mixing processes, thus affecting water quality. Ongoing advances in computer power and improved mathematical modelling of shallow flows have enabled numerical simulation techniques to be used increasingly to model water and sediment flows and mixing processes in open channels.

Lagrangian particle tracking is of great use in investigating dispersion processes in advection-dominated flow fields. The seminal work on chaotic advection of particles is due to Aref ${ }^{[2]}$ who examined the very complicated trajectories of particles in the simple, but abruptly alternating flow field of a pair of blinking vortices. Aref discovered that the particle trajectories altered from periodic to chaotic as the stirring strength increased, and used stroboscopic maps (i.e. snapshots of particle positions at periodic intervals, where the period matches the blinking period of the vortices) to visualise the influence of stirring period on the tendency towards chaos. Kranenburg ${ }^{[3]}$ considered the wind-induced chaotic advection of particles in a closed circular dish-like basin, where the wind direction changed periodically, and the flow field alternated abruptly. Kranenburg's model used a theoretical stream function distribution that fitted the solution to the shallow water equations to estimate the steady-state, wind-induced, depth-averaged velocity field in a circular basin. Although the velocity field is not realistic - depth-averaging obscures three-dimensional flow processes in this case - the progress from periodic to chaotic advection is remarkably similar to that obtained by Aref for the blinking vortex problem, noting that in 
Kranenburg's case the wind strength replaces the stirrer strength as the governing parameter.

There are many examples of the use of particle tracking to study contaminant dispersion in shallow flows. Pearson and Barber ${ }^{[4]}$ applied Lagrangian particle tracking to simulate the dispersion of coastal pollutant by solving an advection-diffusion equation on orthogonal boundary-fitted grids. Weitbrecht et al. ${ }^{[5]}$ used 2-D particle tracking to examine 1D advection-diffusion equation in a river, and validated their model against laboratory measurements of flow in a rectangular open channel with groynes. In a study that is particularly relevant to the present research, Zsugyel et al. ${ }^{[6]}$ utilised particle tracking and meticulous laboratory measurements to investigate mixing processes in the vicinity of a single groyne in a rectangular channel. Zsugyel et al. examined the nonlinear characteristics of the particle trajectories, and presented information on flushing times, particle escape rates, mixing processes (through Lyapunov exponents), and the presence of Lagrangian coherent structures, chaotic saddles, and fractal dimensions. The advection and dispersion of sediment particles is particularly complicated in shallow flow fields near groynes and other hydraulic structures introduced into rivers for control purposes. Field observations, laboratory studies, and numerical simulation all offer insights into advection and mixing processes in open channels ${ }^{[7]}$. The resulting knowledge is useful to hydraulic engineers charged with designing works to control flood flows, protect riverbanks against erosion, and optimize flood protection works.

This paper introduces a coupled numerical solver of the shallow water equations with Lagrangian particle tracking, aimed at studying the chaotic advection of particles near groynes in open channels. The shallow water equations are discretised in space using central differences and the solution marched forward in time using 4thorder Runge-Kutta time integration. The Lagrangian particle-tracking scheme involves integrating the pure advection equation forward in time, again using a 4th-order Runge-Kutta scheme, and is verified against Aref's $\mathrm{s}^{[2]}$ analytical solution for chaotic advection in the flow field of a pair of blinking vortices. The coupled shallow flow and particle-tracking model is then used to simulate chaotic advection and mixing processes in a channel containing a pair of side-wall cavities containing a single groyne, and a rectangular channel containing a pair of groynes. Section 1 presents a short summary of the shallow water equations and the associated solver. Section 2 outlines the particle-tracking methodology. Section 3 presents verification of the particle trajectory model for the case of an abruptly changing flow field described by a pair of blinking vortices. Section 4 investigates particle advection in a pair of adjacent side-wall cavities, and in the vicinity of a pair of groynes in a rectangular channel. Section 5 summarizes the main findings.

\section{Shallow Water Equations}

The two-dimensional shallow water equations apply to nearly horizontal flows in wide, open channels, rivers, and lakes. The shallow water equations may be derived in several ways, including: depth-averaging of the three-dimensional Reynolds-averaged continuity and Navier-Stokes momentum equations obtained from mass and momentum balances across a small control volume [see e.g. Falconer $\left.{ }^{[8]}\right]$; and control volume analysis of elements that extend through the depth, with velocity assumed uniform in the vertical direction [see e.g. Abbott ${ }^{[9]}$ ]. After integration of the Reynolds-averaged continuity and Navier-Stokes momentum equations over the depth from the bed at $z=-h_{s}$ to the free surface at $z=\zeta$, and applying suitable boundary conditions at the bed and free surface, the shallow flow equations are as follows

$$
\begin{aligned}
& \frac{\partial \zeta}{\partial t}+\frac{\partial(U h)}{\partial x}+\frac{\partial(V h)}{\partial y}=0 \\
& \frac{\partial(U h)}{\partial t}+\frac{\partial\left(\beta_{1} U^{2} h\right)}{\partial x}+\frac{\partial\left(\beta_{2} U V h\right)}{\partial y}=f V h-g h \frac{\partial \zeta}{\partial x}+\frac{\tau_{w x}-\tau_{b x}}{\rho}+\frac{1}{\rho} \frac{\partial\left(h T_{x x}\right)}{\partial x}+\frac{1}{\rho} \frac{\partial\left(h T_{x y}\right)}{\partial y},
\end{aligned}
$$

and

$$
\frac{\partial(V h)}{\partial t}+\frac{\partial\left(\beta_{2} U V h\right)}{\partial x}+\frac{\partial\left(\beta_{3} V^{2} h\right)}{\partial y}=-f U h-g h \frac{\partial \zeta}{\partial y}+\frac{\tau_{w y}-\tau_{b y}}{\rho}+\frac{1}{\rho} \frac{\partial\left(h T_{x y}\right)}{\partial x}+\frac{1}{\rho} \frac{\partial\left(h T_{y y}\right)}{\partial y}
$$

in which $U$ and $V$ are the depth-averaged velocity components in the Cartesian $x, y$ directions, $t$ is time, $h$ is total depth ( $\left.h=h_{s}+\zeta\right), f$ is the Coriolis coefficient, $g$ is the acceleration due to gravity, $\rho$ is the density of water,

$\tau_{w x}$ and $\tau_{w y}$ are the wind stress components in the $x$ and $y$-directions, $\tau_{b x}$ and $\tau_{b y}$ are the bed stress components 
in the $x$ and $y$ directions, $T_{x x}, T_{x y}$ and $T_{y y}$ are the effective stresses, and $\beta_{1}, \beta_{2}$ and $\beta_{3}$ are momentum correction factors.

Equations (1), (2), and (3) are discretized using second-order finite differences ${ }^{[7,10,11]}$. The numerical solution is then obtained using an explicit time marching scheme based on the 4th-order Runge-Kutta method. In this case, the grid is rectangular, such that $x=i \Delta x$ and $y=j \Delta y$, where $i=1,2, \ldots i_{\max }$ and $j=1,2, \ldots j_{\max }$ are indices and $\Delta x$ and $\Delta y$ are grid intervals in the $x$ - and $y$-directions. Time is set such that $t=k \Delta t$.

\section{Lagrangian particle tracking scheme}

Lagrangian particle tracking is used to predict the trajectories of tracer particles, with bilinear interpolation providing a representation of the continuous flow field obtained from the discrete results on the numerical grid used by the shallow flow solver ${ }^{[7]}$. In a fluid flow, the advection of a tracer is described by

$u_{p}=\frac{\mathrm{d} x_{p}}{\mathrm{~d} t}, \quad v_{p}=\frac{\mathrm{d} y_{p}}{\mathrm{~d} t}$

where $\left(u_{p}, v_{p}\right)$ are the Cartesian velocity components of a particle located at $\left(x_{p}, y_{p}\right)$. For a passive tracer the particle velocity at a point in space and time is the same as the flow velocity there. The Lagrangian particle tracking-scheme is developed by integrating the advection equations (4) forward in time using a 4th-order RungeKutta time integration method as follows:

$$
\begin{aligned}
& x_{p_{i, j}}^{k+1}=x_{p_{i, j}}^{k}+\frac{1}{6} \Delta t\left(k_{11}+2 k_{12}+2 k_{13}+k_{14}\right) \\
& y_{p_{i, j}}^{k+1}=y_{p_{i, j}}^{k}+\frac{1}{6} \Delta t\left(k_{21}+2 k_{22}+2 k_{23}+k_{24}\right)
\end{aligned}
$$

where

$$
\begin{aligned}
& k_{11}=\frac{\mathrm{d} u_{p}}{\mathrm{~d} t}\left(t, u_{p}\right), \quad k_{21}=\frac{\mathrm{d} v_{p}}{\mathrm{~d} t}\left(t, v_{p}\right), \\
& k_{12}=\frac{\mathrm{d} u_{p}}{\mathrm{~d} t}\left(t+\frac{\Delta t}{2}, u_{p}+\frac{k_{11}}{2}\right), \quad k_{22}=\frac{\mathrm{d} v_{p}}{\mathrm{~d} t}\left(t+\frac{\Delta t}{2}, v_{p}+\frac{k_{21}}{2}\right), \\
& k_{13}=\frac{\mathrm{d} u_{p}}{\mathrm{~d} t}\left(t+\frac{\Delta t}{2}, u_{p}+\frac{k_{12}}{2}\right), \quad k_{23}=\frac{\mathrm{d} v_{p}}{\mathrm{~d} t}\left(t+\frac{\Delta t}{2}, v_{p}+\frac{k_{22}}{2}\right)
\end{aligned}
$$

and

$k_{14}=\frac{\mathrm{d} u_{p}}{\mathrm{~d} t}\left(t+\Delta t, u_{p}+k_{13}\right), \quad k_{24}=\frac{\mathrm{d} v_{p}}{\mathrm{~d} t}\left(t+\Delta t, v_{p}+k_{23}\right)$.

In cases where the velocity field is known analytically as a continuous function, we simply set $\left(u_{p}\left(x_{p}, y_{p}, t\right)\right.$, $\left.v_{p}\left(x_{p}, y_{p}, t\right)\right)=\left(u\left(x_{p}, y_{p}, t\right), v\left(x_{p}, y_{p}, t\right)\right)$ where $u$ and $v$ are the flow velocity components. In cases where the velocity field is computed at discrete points on a grid, the particle velocity is calculated using bilinear interpolation ${ }^{[7]}$. The interpolation process involves three stages. First, the cell containing the particle is identified as $i=i_{\mathrm{p}}$ and $j=j_{\mathrm{p}}$ when $x_{i}<x_{p}<x_{i+1}$ and $y_{i}<y_{p}<y_{i+1}$. We then determine $\left(u_{1}, v_{1}\right)$ at the south side of the cell at a position aligned with $\left(x_{p}, \mathrm{y}_{j p}\right)$ as follows:

$$
u_{1}=u_{i p, j p}+\left(u_{i p+1, j p}-u_{i p, j p}\right)\left(\frac{x_{p}-x_{i p, j p}}{x_{i p+1, j p}-x_{i p, j p}}\right),
$$


$v_{1}=v_{i p, j p}+\left(v_{i p+1, j p}-v_{i p, j p}\right)\left(\frac{x_{p}-x_{i p, j p}}{x_{i p+1, j p}-x_{i p, j p}}\right)$

And we next determine $\left(u_{2}, v_{2}\right)$ at the north side of the cell at a position aligned with $\left(x_{p}, \mathrm{y}_{j p+1}\right)$ as follows:

$u_{2}=u_{i p, j p+1}+\left(u_{i p+1, j p+1}-u_{i p, j p+1}\right)\left(\frac{x_{p}-x_{i p, j p+1}}{x_{i p+1, j p+1}-x_{i p, j p+1}}\right)$,

and

$v_{2}=v_{i p, j p+1}+\left(v_{i p+1, j p+1}-v_{i p, j p+1}\right)\left(\frac{x_{p}-x_{i p, j p+1}}{x_{i p+1, j p+1}-x_{i p, j p+1}}\right)$

The third step involves interpolation in the $y$-direction, giving $\left(u_{p}, v_{p}\right)$ as:

$u_{p}=u_{1}+\left(u_{2}-u_{1}\right)\left(\frac{y_{p}-y_{i p, j p}}{y_{i p, j p+1}-y_{i p, j p}}\right) \quad$,

and

$v_{p}=v_{1}+\left(v_{2}-v_{1}\right)\left(\frac{y_{p}-y_{i p, j p}}{y_{i p, j p+1}-y_{i p, j p}}\right) \quad$.

\section{Model verification for chaotic advection due to a blinking-vortex pair}

To analyze two-dimensional particle advection in the abruptly changing flow field described by a pair of blinking vortices in a closed circular container, Aref ${ }^{[2]}$ assumed a dynamic Hamiltonian system. Aref derived an analytical solution for the integrated particle trajectory from the pure advection equations, and was then able to determine particle patch advection patterns with time, the non-linear dynamics through Poincaré maps, etc., and the trend towards chaotic advection as a vortex strength parameter was increased. Later, Khakhar and Ottino ${ }^{[12-13]}$ repeated much the same exercise for particle advection due to a pair of vortex stirrers in an otherwise infinite expanse of fluid. The present study effectively follows the approach taken by Aref ${ }^{[2]}$. The first vortex is centred at $\left(x_{1}, y_{1}\right)=(-0.5,0)$ and the second vortex at $\left(x_{2}, y_{2}\right)=(0.5,0)$. The blinking vortex model operates by switching on the first vortex, with the second off, for half a cycle during $n T<t<n T+T / 2$ where $n=0,1,2 \ldots$ and $T$ is the prescribed cycle period. The flow field is abruptly switched at $t=n T+T / 2$ so that the second vortex is turned on while the other one is off during the latter half of the cycle when $n T+T / 2<t<(n+1) T$. The process is repeated for $n=0,1,2, \ldots$ until the simulation is complete.

Are ${ }^{[2]}$ defines a dimensionless parameter $\mu$ as follows:

$\mu=\frac{\Gamma T}{2 \pi a^{2}}$

where $T$ is the blinking period, $\Gamma$ is the vortex strength, and $a$ is the distance of the centre of the blinking vortex from the origin. By setting $\Gamma=2 \pi$ and $a=1$, the dimensionless parameter $\mu$ is equal to the period $T$. The choice of value for $\mu$ has a profound effect on the long-term dynamic behaviour of particles. Following Aref ${ }^{[2]}$, we consider blinking vortices located at $\left(x_{1}, y_{1}\right)=(-0.5,0)$ and $\left(x_{2}, y_{2}\right)=(0.5,0)$, and the trajectories of 15 particles whose initial positions are $x_{p}= \pm 0.05, \pm 0.2, \pm 0.35$ with $y_{p}=0$, and $y_{p}=0.1,0.2,0.3,0.4,0.5,0.6,0.7$, $0.8,0.9$ with $x_{p}=0$.

Fig.1 shows stroboscopic maps obtained analytically by Aref ${ }^{[2]}$ as superimposed snapshots of the particle positions taken exactly one cycle apart for different values of $\mu$ for times up to 500T. For $\mu=0.05$, the motion of the particles is generally periodic and observes closed orbits. For $\mu \geq 0.1$, chaotic regions develop close to the 
blinking vortices. As $\mu$ increases further, the chaotic region grows until it fills much of the computational domain. Fig.2 reveals the corresponding stroboscopic maps obtained using the present Lagrangian particletracking model with values of flow velocity determined by interpolation. The results are in reasonable qualitative agreement with those obtained by Aref ${ }^{[2]}$, indicating that the interpolation scheme is working satisfactorily. Next, the particle positions are considered after different numbers of blinking vortex cycles where an initial square array of 6400 particles is divided into four quadrants, each of different colours for $\mu=1$. The Lagrangian particletracking results in Fig.3 show that mixing is more rapid and widespread when a higher value of $\mu$ is considered. Transition to chaos is evident in regions near the stirrers, with whorls and tendrils evident at the periphery.
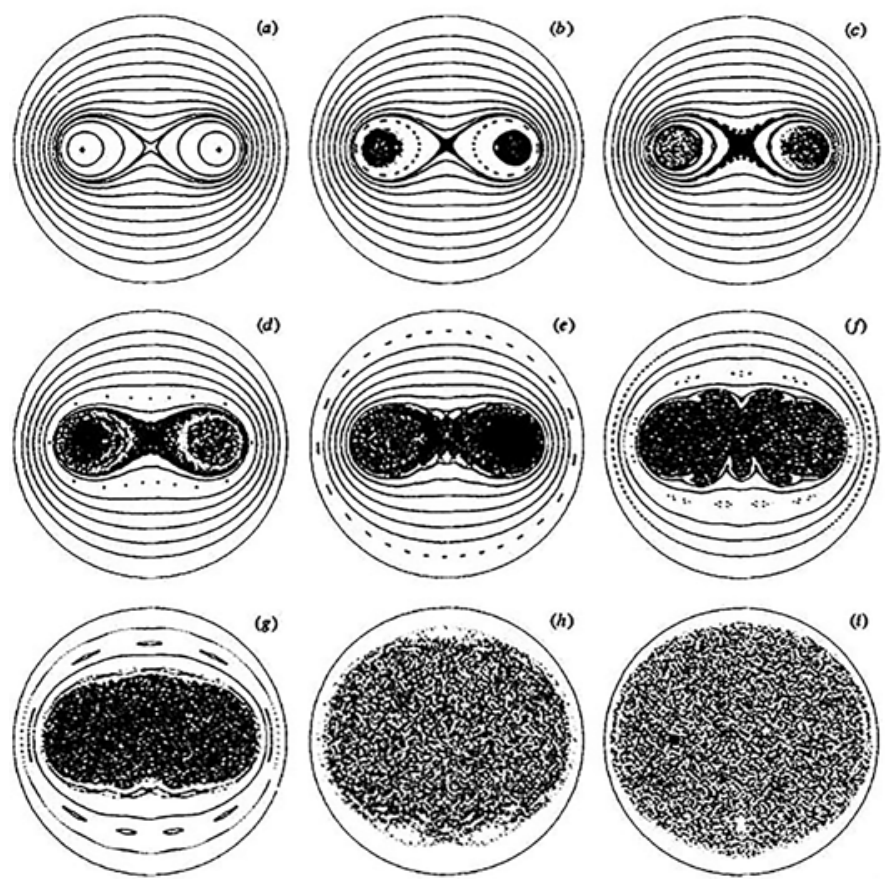

Fig.1 Analytically predicted stroboscopic maps of particles in the flow field induced by a pair of blinking vortices (Aref, 1984): (a) $\mu=0.05$, (b) $\mu=0.1$, (c) $\mu=0.125$, (d) $\mu=0.15$, (e) $\mu=0.2$, (f) $\mu=0.35$, (g) $\mu=0.5$, (h) $\mu=1.0$, and (i) $\mu=1.5$ 
(a)

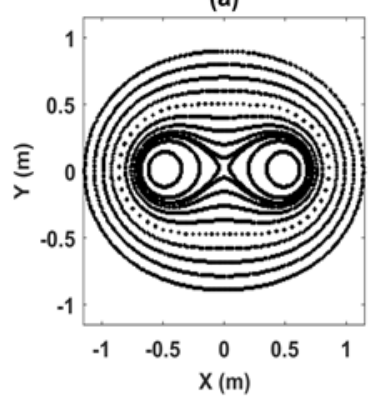

(d)

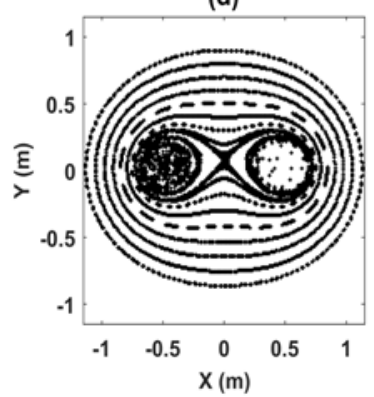

(g)

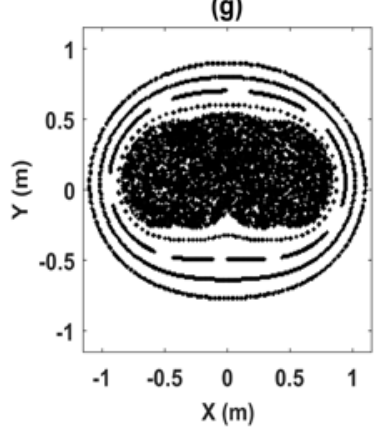

(b)

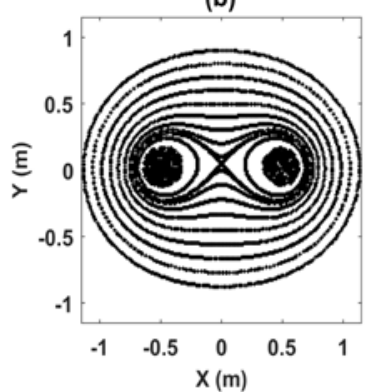

(e)

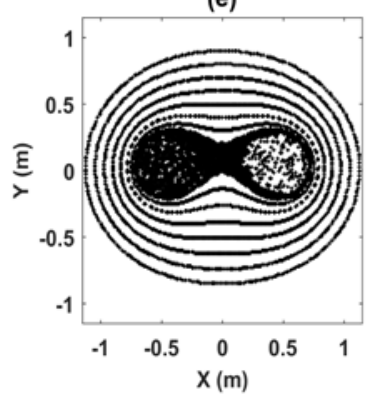

(h)

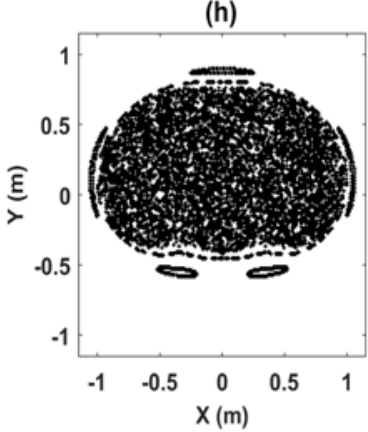

(c)

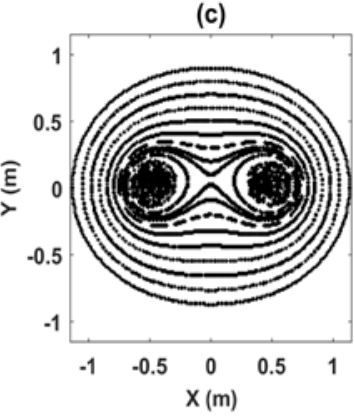

(f)

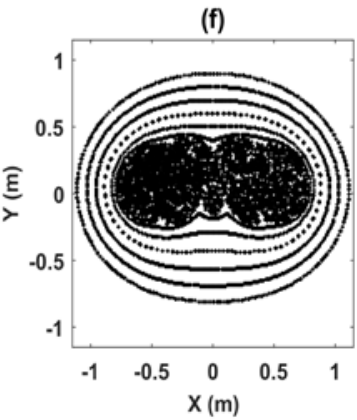

(i)

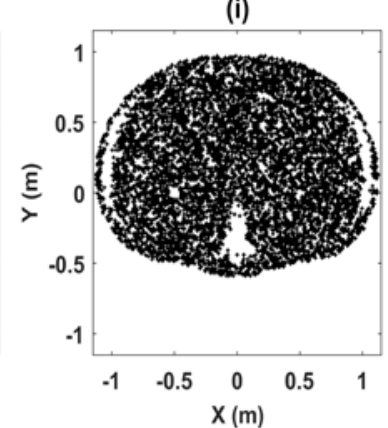

Fig.2 Numerically predicted stroboscopic maps of particles in the bilinear interpolated flow field induced by a pair of blinking vortices: (a) $\mu=0.05$, (b) $\mu=0.1$, (c) $\mu=0.125$, (d) $\mu=0.15$, (e) $\mu=0.2$, (f) $\mu=0.35$, (g) $\mu=0.5$, (h) $\mu=1.0$, and (i) $\mu=1.5$ 
(a)

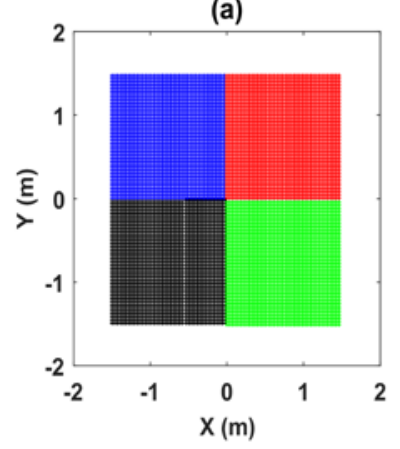

(d)

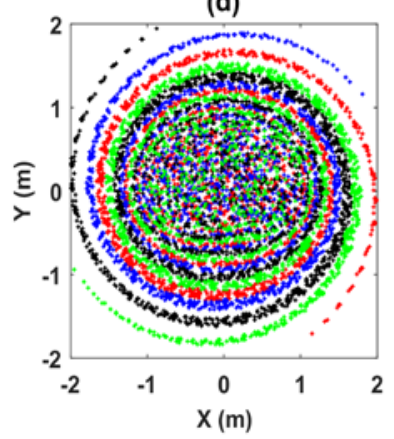

(b)

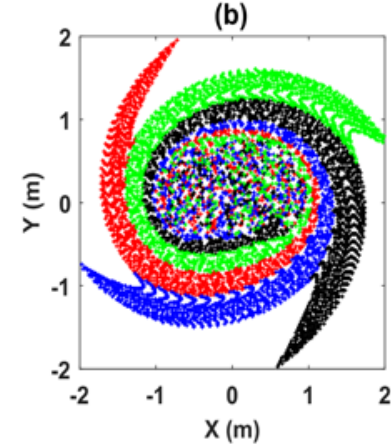

(e)

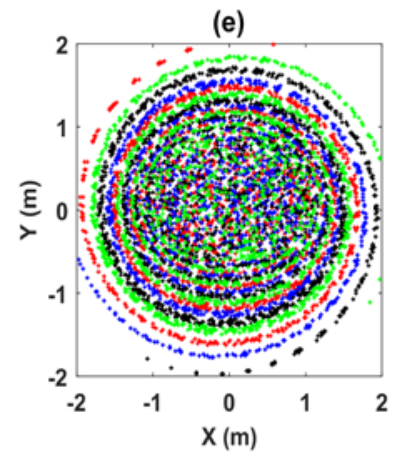

(c)

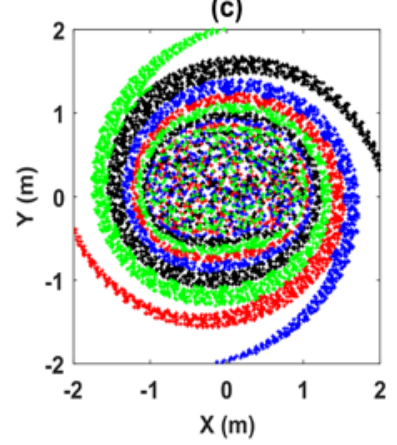

(f)

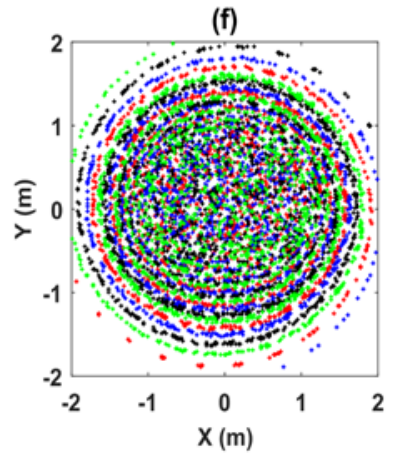

Fig.3 Numerically predicted particle positions in the bilinear interpolated flow field induced by a pair of blinking vortices when $\mu=1.0$ at times: (a) $t=0.0 T$ (b) $t=5.0 T$, (c) $t=10.0 T$, (d) $t=20.0 T$, (e) $t=30.0 T$, (f) $t=40.0 T$

\section{Advection of particles in the vicinity of a groyne}

\subsection{Particle advection in a pair of adjacent side-wall cavities}

We now consider the flow past a side-wall expansion containing an idealized groyne in a rectangular open channel, following previous laboratory measurements by Tuna and Rockwell[14] who examined selfsustained resonant oscillations due to phase shifts in standing wave modes in the cavities and undulations in the separated shear later, in flow past a pair of adjacent side-wall cavities. The shallow flow solver is used to reproduce the flow conditions in a qualitatively similar configuration to that considered by Tuna and Rockwell. In our cases, the main channel is $700 \mathrm{~mm}$ long and $475 \mathrm{~mm}$ wide, with flow entering from the left hand end of the open channel. After an initial reach of $200 \mathrm{~mm}$, the channel widens abruptly into a side-wall expansion of length $300 \mathrm{~mm}$ and additional width $225 \mathrm{~mm}$. The expansion zone contains a groyne of $10 \mathrm{~mm}$ width that projects outward $225 \mathrm{~mm}$, perpendicular to the channel wall. The groyne effectively divides the region of the side-wall expansion into a pair of adjacent side-wall cavities. After the side-wall expansion region, the channel again abruptly becomes $475 \mathrm{~mm}$ wide. The mean water depth is $38 \mathrm{~mm}$. We consider two cases, where the inlet velocity, $U_{\mathrm{I}}$, is set to $273 \mathrm{~mm} / \mathrm{s}$ and $475 \mathrm{~mm} / \mathrm{s}$, respectively.

Fig.4 shows a typical set of stream function contours obtained by Tuna and Rockwell from time-averaged PIV velocity measurements for flow past a pair of adjacent side-wall cavities. In the present work, the numerical model is based on a uniform 280 by 280 grid that covers an overall computational domain, measuring $700 \mathrm{~mm}$ by $700 \mathrm{~mm}$, which includes the open channel and side-wall cavities. For $U_{I}=273 \mathrm{~mm} / \mathrm{s}$, the eddy viscosity is set to $9.992 \mathrm{~mm}^{2} / \mathrm{s}$, and the time step $\Delta t=0.01 \mathrm{~s}$. For $U_{I}=475 \mathrm{~mm} / \mathrm{s}$ the eddy viscosity is $17.264 \mathrm{~mm}^{2} / \mathrm{s}$, and the time step $\Delta t=0.001 \mathrm{~s}$. In both cases, the overall simulation time is $20 \mathrm{~s}$. It should be noted that, after grid convergence was checked, the time step was selected so that the Courant number remained below unity. Fig.5 displays the steady state stream function contour results for the two cases considered using the present shallow flow solver. The contours indicate that larger vortices are produced in the two side-wall cavities at the higher value of inlet velocity, as would be expected. The present results are in qualitative agreement with those of Tuna and Rockwell $^{[14]}$ (Fig.5), implying that the shallow flow solver gives a potentially useful representation of flowinduced vortices in adjacent side-wall cavities separated by a single groyne. 


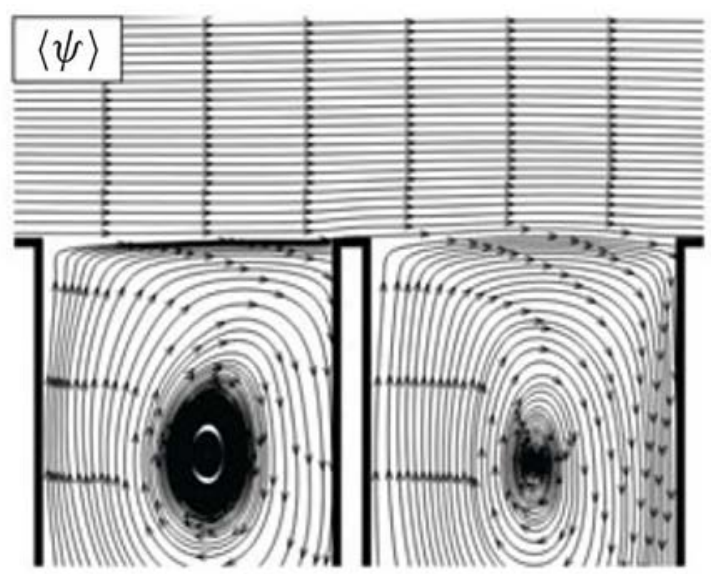

Fig.4 Time-averaged stream function contours during a transverse mode of a gravity standing wave in a pair of adjacent side-wall cavities in open channel flow obtained from PIV measurements by Tuna and Rockwell ${ }^{[14]}$

(a)

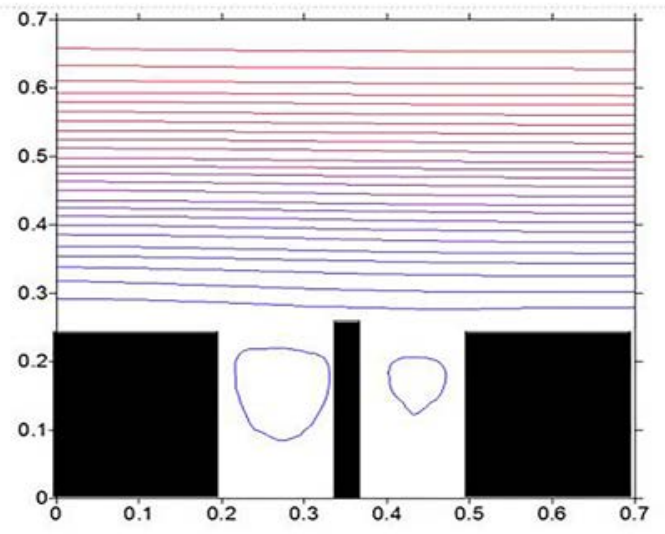

(b)

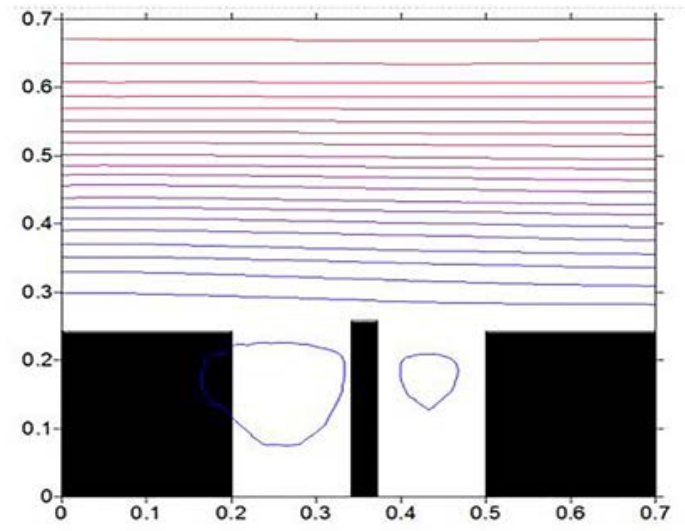

Fig.5 Stream function contours for open channel flow past a pair of adjacent side-wall cavities for: (a) $U_{1}=273 \mathrm{~mm} / \mathrm{s}$, and (b) $U_{2}=$ $475 \mathrm{~mm} / \mathrm{s}$

The coupled shallow flow and particle-tracking model is then used to simulate advection and mixing processes in the side-wall cavities divided by the groyne, owing to the presence of the two recirculation zones, one in each cavity. To visualize particle mixing, the space within each cavity is filled with four layers of particles, the layers containing red, blue, purple and green particles, respectively. In this study, 54000 particles are released, uniformly distributed throughout the cavities. Here, the time step used by the Lagrangian particle tracker is $0.5 \mathrm{~s}$. Fig.6 shows snapshots of the particle positions at different times after their release, for a case where the inlet velocity is $273 \mathrm{~mm} / \mathrm{s}$. At first, the northern green and purple layers undulate. The undulations then develop standing waveforms which then curl over and form whorls. The blue layer gradually gets pulled towards the main stream, and exhibits cuspate peaks. 

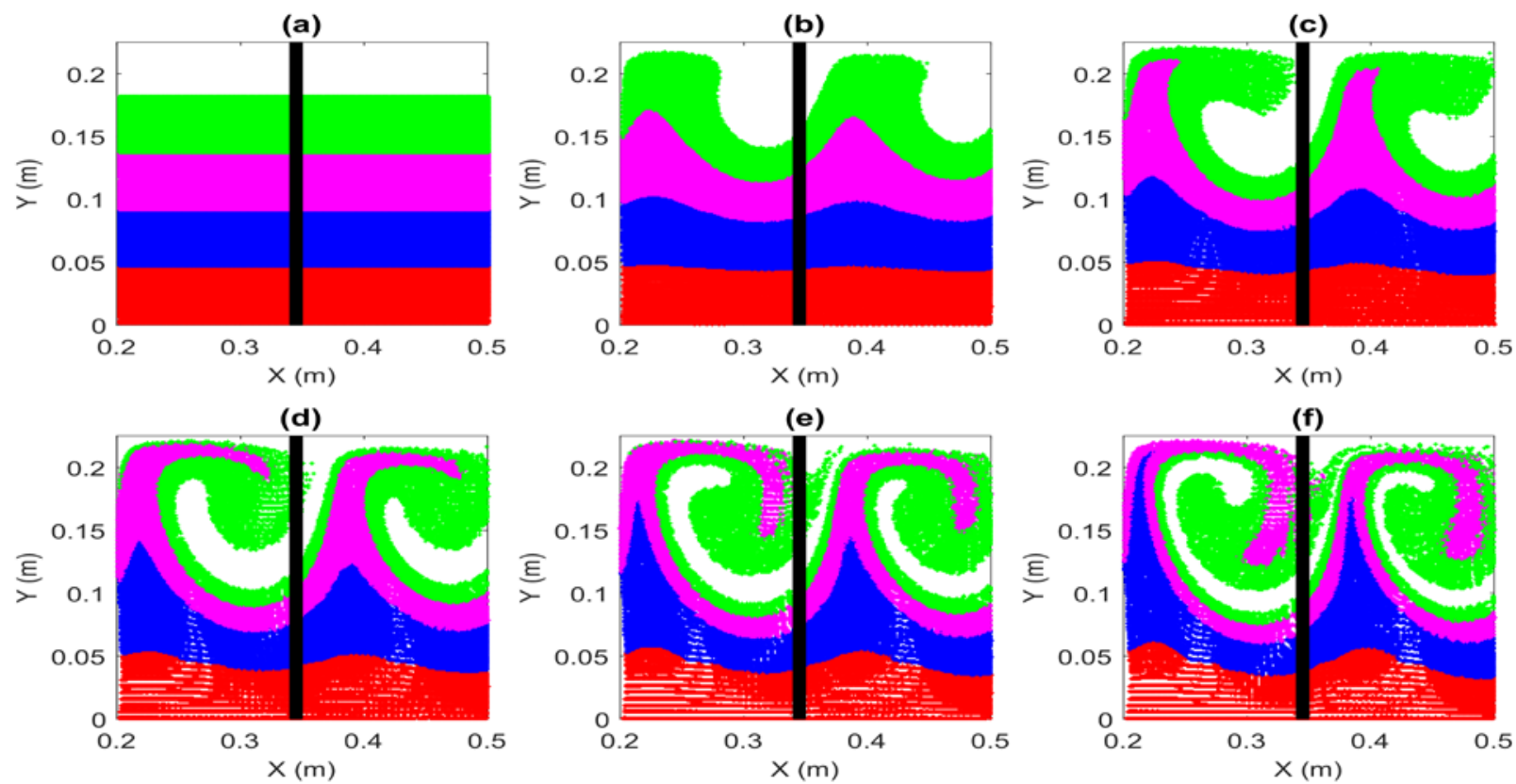

Fig.6 Particle advection in a pair of adjacent side-wall cavities for $U_{1}=273 \mathrm{~mm} / \mathrm{s}$ at times: (a) $t=0 \mathrm{~s}$, (b) $t=10 \mathrm{~s}$, (c) $t=20 \mathrm{~s}$, (d) $t=$ $30 \mathrm{~s}$, (e) $t=40 \mathrm{~s}$, (f) $t=50 \mathrm{~s}$
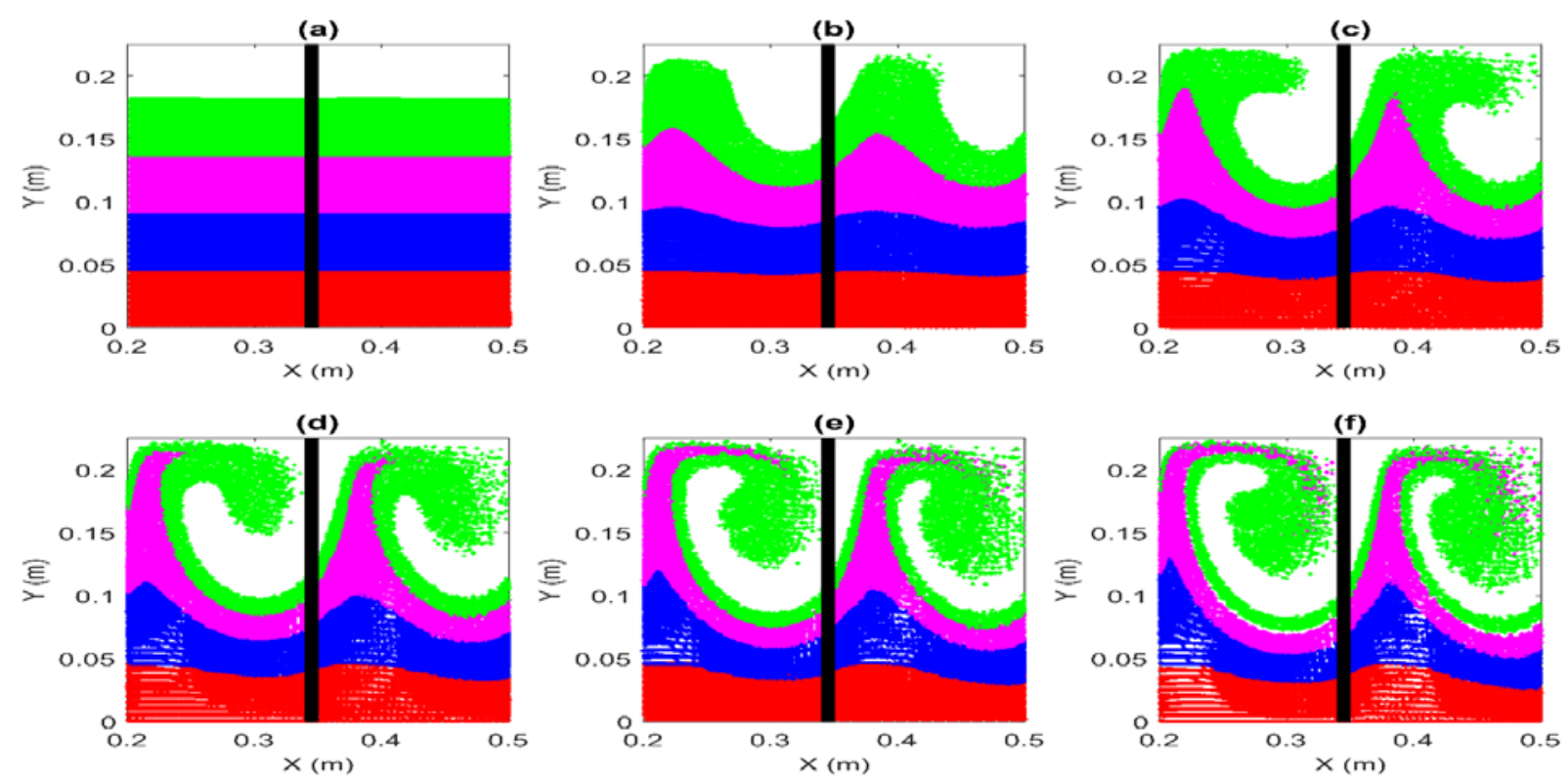

Fig.7 Particle advection in a pair of adjacent side-wall cavities for $U_{2}=475 \mathrm{~mm} / \mathrm{s}$ at times: (a) $t=0 \mathrm{~s}$, (b) $t=5 \mathrm{~s}$, (c) $t=10 \mathrm{~s}$, (d) $t=$ $15 \mathrm{~s}$, (e) $t=20 \mathrm{~s}$, (f) $t=25 \mathrm{~s}$

As can be seen in Fig.7, similar results are obtained for the higher inlet velocity of $475 \mathrm{~mm} / \mathrm{s}$, though the roll up of the magenta and green layers takes place more quickly.

\subsection{Mixing of particles in a rectangular channel containing a pair of parallel groynes}

The coupled shallow water and particle-tracking model is now used to simulate particle advection in a rectangular open channel containing a pair of groynes. Here, the channel has plan dimensions of $3600 \mathrm{~m}$ x $300 \mathrm{~m}$. The inlet velocity is $0.5 \mathrm{~m} / \mathrm{s}$, Reynolds number is 100 , water depth is $1 \mathrm{~m}$, and eddy viscosity is $0.5 \mathrm{~m}^{2} / \mathrm{s}$. The time step is $5 \mathrm{~s}$, satisfying the Courant criterion, and the simulation time to flow steady state is $4000 \mathrm{~s}$. To study particle 
mixing in the vicinity of the groynes, the particle-tracking model is then used to simulate trajectories of an initially rectangular array of 8000 particles, with five colour bands, which is released upstream of the first groyne. Fig. 8 shows the advected distributions of the particles at intervals of $200 \mathrm{~s}$ after the initial release into the steady state flow. It can be seen that the red particles become stretched along a tendril, with blue, magenta, green, and yellow particles becoming more spread out as time progresses from $t=0 \mathrm{~s}$ (the time of particle release) to $t=1000$ s. Fig.10 shows the results obtained in the same rectangular channel containing two groynes, this time with an array of 54000 particles that initially entirely fill the domain; the snapshots indicate the evolving particle distributions obtained with time. Here, the yellow particles become the most separated over the long term, being advected by the main current in the channel. The red particles are initially the least separated because many become temporarily trapped in the recirculation zones.

(a)

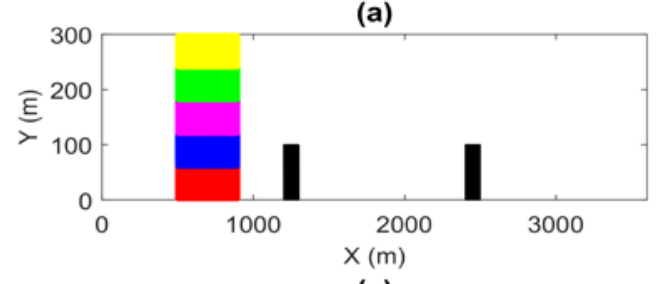

(c)

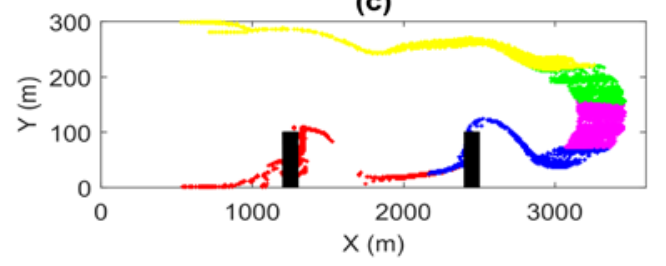

(e)

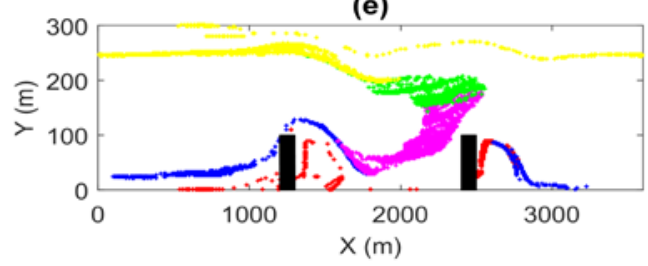

(b)

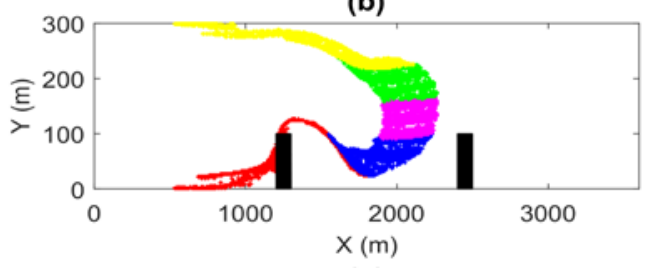

(d)

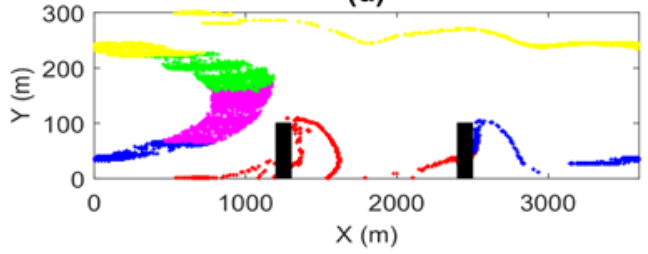

(f)

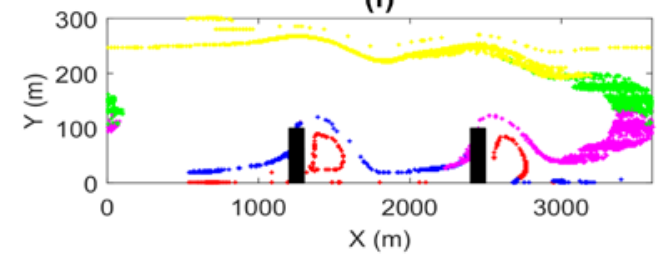

Fig.8 Mixing of an array of 8000 particles in an open rectangular channel containing two groynes at times: (a) $t=0 \mathrm{~s}$, (b) $t=200 \mathrm{~s}$, (c) $=400 \mathrm{~s}$, (d) $t=600 \mathrm{~s}$, (e) $t=800 \mathrm{~s}$, and (f) $t=1000 \mathrm{~s}$

(a)

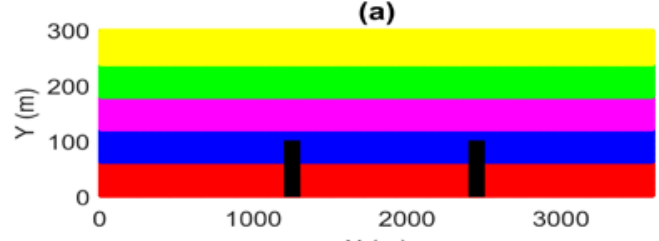

$\mathrm{x}(\mathrm{c})$

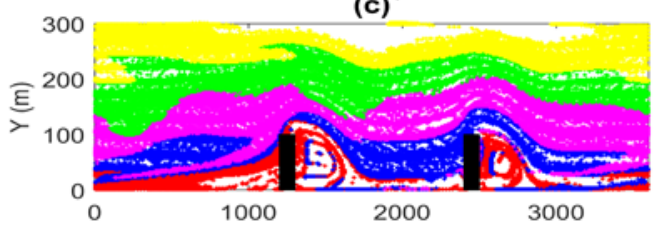

$x(\mathrm{e})$

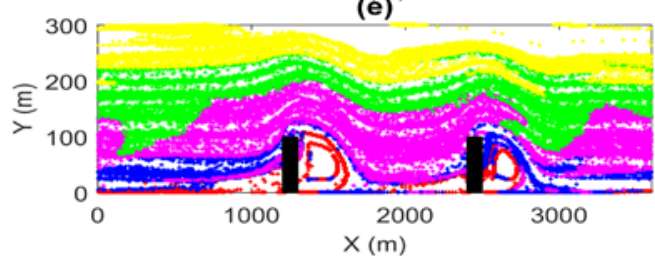

(b)

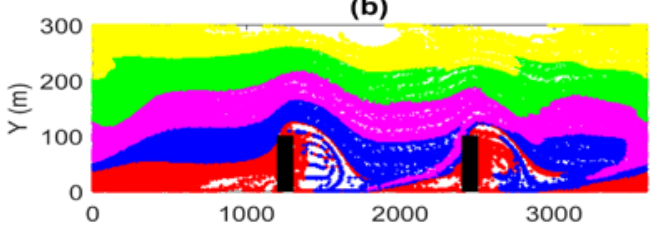

$x($ (d)
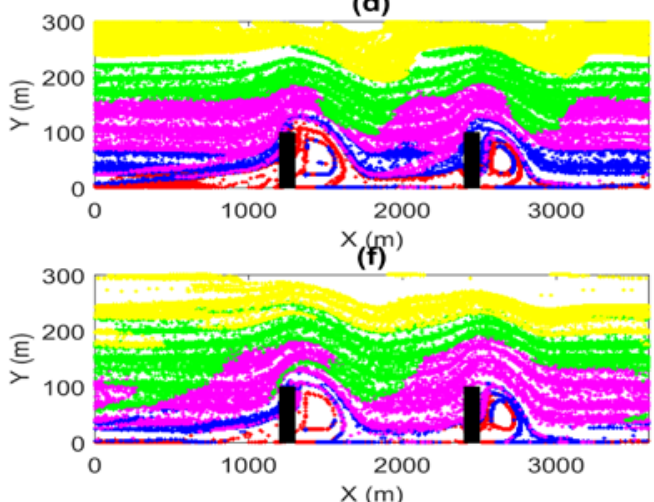

Fig.9 Mixing of an array of 54000 particles in an open rectangular channel containing two groynes at times: (a) $t=0 \mathrm{~s}$, (b) $t=200 \mathrm{~s}$, (c) $t=400 \mathrm{~s}$, (d) $t=600 \mathrm{~s}$, (e) $t=800 \mathrm{~s}$, and (f) $t=1000 \mathrm{~s}$ 


\section{Conclusions}

A coupled shallow water solver and Lagrangian particle-tracking scheme has been constructed using $2^{\text {nd }}$ order finite differences in space and $4^{\text {th }}$ order Runge-Kutta integration in time. The particle-tracking algorithm was verified for periodic and chaotic advection in the alternating flow field of a pair of blinking vortices. Close agreement was obtained with analytical results presented in the literature by Aref ${ }^{[2]}$; it should be noted that the present particle tracking model used an interpolated discretized velocity field instead of the continuous analytical velocity field. As the stirrer strength $\mu$ increased from 0.05 to 1.5 , the chaotic region grew until it filled much of the computational domain, in accordance with Aref's findings. The coupled shallow water flow and Lagrangian particle-tracking model was further validated against laboratory data for open channel flow past a pair of adjacent side-wall cavities obtained by Tuna and Rockwell ${ }^{[14]}$. Results were presented for the advection of different coloured tracer particles in the region of the side-wall cavities and it was found that the majority of particles remained trapped in the side-wall cavities, with a tendency for the particles to roll up into a single whorl that filled each cavity. Mixing of particles in a rectangular channel containing a pair of parallel groynes, oriented perpendicular to the lateral walls, has also been simulated using the coupled shallow flow and particle-tracking model. The use of different colour bands of particles highlighted particle trapping that occurred immediately downstream of each groyne within the recirculation zones, with the majority of particles passing the ends of the groynes (in the main through-flow stream).

\section{Acknowledgements}

The first author is grateful to the University of Edinburgh which partly funded this research.

\section{References}

[1] Rutherford, J. C. Handbook on Mixing in Rivers [R]. Water \& Soil Miscellaneous Publication No. 26, 1981, National Water \& Soil Conservation Organisation, New Zealand, 60 pp, ISSN 0110-4705.

[2] Aref, H. Stirring by chaotic advection [J]. Journal of Fluid Mechanics, 1984, 143: 1-21.

[3] Kranenburg, C. Wind-driven chaotic advection in a shallow model lake [J]. Journal of Hydraulic Research, 1992, 30 (1): 29-46.

[4] Pearson, R. V., Barber, R. W. Modelling depth-integrated coastal pollution using a Lagrangian particle technique [J]. Transactions on Ecology and the Environment, 1998, 17: 233-240.

[5] Weitbrecht, V, Uijttewaal, W.S.J., Jirka, G. H. 2-D Particle tracking to determine transport characteristics in rivers with dead zones [C]. Int. Symp. on Shallow Flows, Delft, 2003, 1-8.

[6] Zsugyel, M, Tél, T, Józsa, J. Numerical investigation of chaotic advection past a groyne based on laboratory flow experiment [J]. Advances in Water Resources, 2014, 71: 81-92.

[7] Jalali, M.M. Curvilinear Shallow Flow and Particle Tracking Model for a Groyned River Bend [D]. PhD Thesis, The University of the Edinburgh, UK, 2016:1-184.

[8] Falconer, R.A. (1993) An introduction to nearly-horizontal flows. Chapter 2, Coastal, Estuarial and Harbour Engineer's Reference Book [M]. Edited by M.B. Abbott and W.A. Price, Chapman \& Hall, London, 27-36.

[9] Abbott M. B, Computational Hydraulics: Elements of the Theory of Free Surface Flows [M]. Pitman Advanced Publishing Program: London, 1979, 324 p.

[10] Fletcher, C. Computational Techniques for Fluid Dynamics 1, Fundamental and General Techniques, 2nd Edition [M]. Springer-Verlag Berlin Heidelberg, 1998, 401 p.

[11] Abbott, M. B, Basco, D. R. Computational Fluid Dynamics - An Introduction for Engineers [M]. Longman Scientific and Technical/John Wiley and Sons, Harlow, England/New York, 425, 1989, 439 p.

[12] Khakhar, D. V., Ottino, J. M. Fluid mixing (stretching) by time periodic sequences for weak flows [J]. The Physics of Fluids, 1986, 29 (11): 3503-3505.

[13] Khakhar, D. V, Rising, H, Ottino, J. M. Analysis of chaotic mixing in two model systems. [J] Journal of Fluid Mechanics, 1986, 172: 419-451.

[14] Tuna, B. A., Rockwell, D. Self-sustained oscillations of shallow flow past sequential cavities. [J] Journal of Fluid Mechanics, 2014, 758: 655-685. 